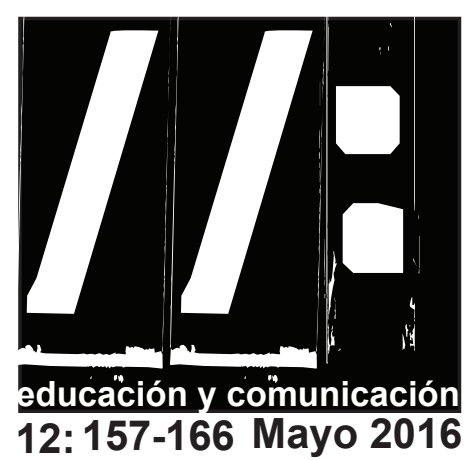

Resumen:

\section{EL TEMA 'MIGRACIONES' EN MATERIALES DIDÁCTICOS Y LA FORMACIÓN INTERCULTURAL DEL PROFESORADO DE LENGUA ESPAÑOLA}

The theme 'migrations' in teaching materials and the intercultural education of spanish language teachers

\author{
Doris Cristina Vicente da Silva Matos \\ Grupo de Investigación: DInterLin: \\ Diálogos Interculturais e Linguísticos \\ Departamento de Letras Estrangeiras \\ Universidade Federal de Sergipe (Brasil) \\ E.mail: profadoris_ufs@yahoo.com.br
}

Este estudio invita a la discusión de prácticas educacionales sobre el desarrollo de la formación del profesorado de lengua española en Brasil bajo una perspectiva intercultural, de manera que se entiendan las oportunidades que ofrece la diversidad en beneficio de la sociedad y se pueda aprender a afrontar sus desafíos en la enseñanza de lenguas. Para ello, parto de una experiencia de elaboración de materiales didácticos en un contexto de formación inicial del profesorado de español con el tema 'migraciones' y verifico si son capaces de producir materiales que promuevan un aprendizaje crítico por parte de los alumnos, basado en una perspectiva intercultural. Los resultados apuntan a la necesidad de fomentar desde el inicio de la titulación el interés por la investigación académica a través de proyectos que involucren el profesorado con la rutina de la escuela, contribuyendo significativamente a la práctica pedagógica de los futuros profesores.

Palabras clave: Formación del profesorado, Interculturalidad; Materiales didácticos.

Abstract:

This study invites for a discussion of educational practices about the brazilian spanish teachers formation development in Brazil, under an intercultural perspective, in order to understand the opportunities that offer a diversity in benefit of the society and learn to affront its challenges in the languages teaching. For this, I begin from an experience of teaching materials preparing in a context of initial formation of spanish teachers on the "migration" theme and I check if they are capable of producing materials that promotes a critical learning on the students, based on an intercultural perspective. The results points for the necessity of fomenting, since the beginning of the graduation, the interest for the academic investigation through the projects that involves the teachers with the school routine, significantly contributing for the pedagogic practice of the future teachers.

Key words: Teacher education, Intercultural,Teaching materials.

Recibido: 16/11/2015 Revisado: 09/02/2016 Aceptado: 30/03/2016 Publicado: 01/05/2016 


\section{IIA Interculturalidad, educación y comunicación}

\section{Consideraciones iniciales}

Q $\mathrm{n}$ la sociedad que vivimos hay una necesidad de Eformación crítica del profesorado para que ellos estén atentos a las exigencias del mundo contemporáneo, por eso es urgente reflexionar sobre los caminos que esta formación debe seguir. Los currículums de los Cursos de Letras, titulación que capacita para que uno sea profesor de lengua española en diversos contextos, principalmente en escuelas, necesitan englobar conocimientos que permitan al futuro profesor ser capaz de planear sus clases con autonomía y de conformidad con las actuales propuestas de abordaje para la enseñanza de lenguas. Entre estos abordajes defiendo el trabajo a partir de una perspectiva intercultural, tanto en su ejecución en las escuelas como en la formación del profesorado en los Cursos de Letras. Toda actividad didáctico pedagógica debe pasar por una etapa indispensable antes de ser puesta en práctica, a esta etapa la denominamos planteamiento y la elección de trabajar con el planteamiento para la elaboración de materiales didácticos ocurrió por el hecho de percibir en mi práctica, como profesora de prácticas de español en la Universidad Federal de Sergipe (Brasil), la dificultad que muchos alumnos presentan a la hora de planear una clase. Muchas preguntas surgen en este momento, como por ejemplo: ¿Por dónde empezar? ¿Trabajo gramática? ¿Qué tema es adecuado para este grupo de alumnos? ¿Qué puede motivar a mis alumnos? ¿Cómo presentar cuestiones culturales? ¿Qué género textual es más adecuado?, y muchas otras.

Esas inquietudes me motivaron a investigar en el área de la formación del profesorado la elección del material didáctico ya que la considero de fundamental importancia para que el planteamiento de las clases tenga grandes oportunidades de obtener éxito. Claro está que otros factores van a influir en el ambiente de clase, como los propios alumnos, la atmósfera de clase o cualquier otro factor interno o externo que pueda influir en la ejecución de la clase. Mi parte es, aquí, la del material didáctico y el camino que el profesor en formación siguió para elaborarlo, pautado en el carácter formativo e intercultural de la lengua extranjera. Parto del presupuesto de que todo material trae de forma explícita o implícita la concepción filosófica, teórica y didáctico metodológica de quien lo produjo, lo que va a reflejar en su uso en los contextos de enseñanza y aprendizaje (Almeida Filho, 2013).

Con esta breve discusión, este trabajo pretende establecer relaciones entre la formación de profesores de español en Brasil, a partir de una perspectiva intercultural y la elaboración de materiales didácticos. Pretendo provocar reflexiones sobre la necesidad de producción de materiales que posibiliten la construcción de acciones pedagógicas orientadas por una postura crítica, política y comprometida con prácticas sociales interculturales y, para ello, parto de una experiencia de elaboración de materiales didácticos en contexto de formación inicial del profesorado de español en Brasil con el tema 'migraciones' y verifico si ellos son capaces de producir materiales que promuevan un aprendizaje crítico por parte de los alumnos, basado en una perspectiva intercultural.

\section{Interculturalidad y materiales didácticos}

El mercado de materiales didácticos de lengua extranjera, más específicamente de español, sufrió lo que podemos calificar como boom en Brasil a partir del 


\section{1/: El tema "migraciones" en materiales didácticos y la formación intercultural del profesorado de lengua española}

año 2008, con la inclusión del componente curricular de lengua extranjera moderna en el Programa Nacional del Libro Didáctico (PNLD), a través del cual fue posible la inscripción de materiales didácticos de inglés y español para adopción en las escuelas públicas de todo el país. Costa (2011: 6), al dimensionar el tipo de material demandado por el PNLD, indica que es:

«[...] un libro concebido metodológicamente de modo que los textos y las actividades posibiliten el aprendizaje efectivo de la lectura, escritura, audición y habla en LE, en la escuela, en una perspectiva crítica y reflexiva, contribuyendo así a la formación de la ciudadanía»

El PNLD es uno de los programas del 'Fundo Nacional de Desenvolvimento da Educação' (FNDE), autarquía federal vinculada al Ministério de Educación (MEC), que tiene como finalidad la ejecución de políticas para el desarrollo de programas y proyectos que busquen la mejora de la educación en nuestro país. Las primeras convocatorias, de 2008 y 2009, fueron publicadas, respectivamente, para el PNLD de la Enseñanza Fundamental (EF) 2011 y para el PNLD de la Enseñanza Media (EM) 2012.

Tras la evaluación de las colecciones de lengua extranjera inscritas y que estaban de acuerdo al dispuesto en la convocatoria, en el PNLD de la EF 2011, de las 37 colecciones -11 de español y 26 de inglés-, solamente un total de 4 -dos de cada lengua- fueron aprobadas. Ya en el PNLD de la EM 2012, de las 32 colecciones -12 de español y 20 de inglés-, un total de 13 -3 de español y 10 de inglés- fueron seleccionadas. Las convocatorias siguientes, de 2011 y 2013, fueron publicadas respectivamente para el PNLD de la EF 2014 y para el PNLD de la EM 2015. El primero, PNLD de la EF 2014 contó con 36 colecciones inscritas - 15 de español y 21 de inglés- de ese total, solamente 5 colecciones fueron aprobadas, con 2 de español y 3 de inglés. El PNLD de la EM 2015 contó con 27 colecciones inscritas -13 de español y 14 de inglés- de esas fueron aprobadas 6 colecciones, 2 de español y 4 de inglés. Actualmente el PNLD del EF 2017 se encuentra en ejecución, motivo por el cual aún no es posible obtener más informaciones.

La inclusión del componente curricular de lengua extranjera en el PNLD es un avance en política educacional, pues se realiza una gran inversión financiera para que los libros didácticos sean distribuidos en las escuelas públicas de nuestro país. Inversión que prima por la calidad de las obras seleccionadas, de manera que los alumnos y profesores brasileños puedan tener en sus manos un material que sea una herramienta importante para la formación de ciudadanos críticos. Sobre el proceso de revisión de los libros didácticos del PNLD, Silva (2008: 169) explica que:

«[...] se realiza con ayuda de técnicos, maestros y doctores de varias partes de Brasil, se examinan los libros del mercado editorial, observando si ellos vehiculan conceptos correctos, si tienen una orientación metodológica moderna, si educan a los alumnos para la ciudadanía etc., en fin, si están mínimamente de acuerdo con los principios que deben guiar la enseñanza en Brasil»

Y, ¿cuáles serían los principios que deben guiar la enseñanza en Brasil, especialmente de la lengua española? Al tomar la enseñanza de una lengua extranjera como espacio de encuentro y diálogo entre culturas, las relaciones establecidas en el contexto escolar podrán tanto promover como alejar ese encuentro. Cuando hablo de cultura en el aula de lengua extranjera, no me refiero a la celebración folclórica de la diversidad de países o naciones a través de sus características 


\section{II: Interculturalidad, educación y comunicación}

estereotipadas (Canclini, 2006). En un mismo país o nación hay el embate de más de una cultura, si reconocemos la diversidad cultural y social como elementos constitutivos de las sociedades modernas. Las sociedades son, en su esencia, culturalmente heterogéneas, y reconocer y valorar esta característica son pasos para una mirada intercultural centrada en el desarrollo de las personas para la práctica de la ciudadanía.

A pesar de este avance, es necesario que los profesores estén preparados para trabajar con materiales que aborden cuestiones diversas y no solamente el clásico estudio de formas gramaticales descontextualizadas y pautadas en la memorización de reglas en sus unidades. Creo que un buen material necesita un profesor bien preparado para que sus posibilidades pedagógicas sean explotadas al máximo y él/ella sea capaz de sanar los huecos que puedan existir en tales materiales, ya que nuestro país posee dimensiones continentales y un único material no será probablemente capaz de dar cuenta de las diversidades geográficas/ regionales en que vivimos, además de la diversidad cultural hispánica.

Brasil y los países hispánicos terminan siendo vistos de forma homogénea en muchas publicaciones editoriales y, de esa manera, la diversidad cultural sufre un apagamiento en su constitución. Siendo así, los libros didácticos, muchas veces, limitan el ejercicio de la crítica, tendiendo al prejuicio y al reduccionismo, lo que no está de acuerdo con el fomento a la diversidad cultural y a los principios de una práctica intercultural. Aguado (2003: 129) apunta que el contenido de los recursos educativos puede tener influencia en las actitudes de los alumnos sobre sí mismos y sobre los otros, ya que "todos los materiales pedagógicos utilizados son mediadores culturales decisivos, pues son la explicitación del qué y del cómo son valorados los elementos culturales que serán transmitidos".

A partir de esta necesidad detectada en la formación de los profesores de lenguas, surgió la idea de elaborar un material en conjunto con alumnos del Curso de Letras con habilitación en lengua española de la Universidad Federal de Sergipe; sin embargo, solo su existencia no es capaz de fomentar y asegurar que el profesor actúe con una perspectiva intercultural:

«Ser y actuar de modo culturalmente sensible a los sujetos participantes del proceso de aprendizaje exige de aquellos que planean cursos, elaboran materiales didácticos y enseñan una LE/L2, mucho más que la simple elección de contenidos apropiados. Exige esfuerzo especial para hacer con que las experiencias de enseñar y aprender, más que medios de alcanzar competencia comunicativa en la nueva lengua-cultura, sean un medio de construir, a través del diálogo entre culturas, la interacción entre sujetos-mundos diferentes y la construcción de nuevos conocimientos y experiencias surgidos a partir de ese encuentro" (Mendes, 2004: 222)»

Al actuar como investigadora, necesité definir algunos criterios para la elaboración de las unidades didácticas, y el primero de ellos fue la temática que sería desarrollada por los participantes de la investigación. Al defender la perspectiva intercultural como medio de mediación en clase, opté por un tema amplio que pudiera abarcar un gran rol de subtemas, que fue definido como: Identidades socioculturales en clases de español como lengua extranjera.

La cuestión de las identidades es muy discutida tanto en ámbito académico cuanto en la sociedad en general. Moita Lopes (2003) aborda el tema desde un enfoque socioconstructivista, apuntando que los objetos sociales están en constante reconstrucción y 


\section{1/: El tema "migraciones" en materiales didácticos y la formación intercultural del profesorado de lengua española}

que los cambios ocurrirán a partir de las interacciones trabadas socialmente, de esa manera, las identidades sociales son fragmentadas, contradictorias en ebullición y en constante flujo. De esta perspectiva es posible comprender los recurrentes cuestionamientos de quiénes somos en la sociedad y en el mundo en que vivimos:

«Hay en las prácticas cotidianas que vivimos un cuestionamiento constante de modos de vivir la vida social que viene afectando la comprensión de la clase social, del género, de la sexualidad, de la edad, de la raza, de la nacionalidad, etc., en resumen, de quiénes somos en la vida social contemporánea"(Moita Lopes, 2003: 15)»

Rajagopalan (2003: 71) corrobora este pensamiento al indicar que "en cualquier momento dado, las identidades son adaptadas y adecuadas a las nuevas circunstancias que van surgiendo". De esa manera, la escuela necesita reestructurarse internamente para crear el diálogo necesario entre los sujetos/ alumnos y esa realidad a la cual están sometidos, ya que los significados construidos en clase poseen relación directa con la definición de las identidades sociales de los alumnos.

Al elegir el tema generador, tenía en mente el desarrollo de unidades didácticas que expusieran los cambios que ocurren en nuestra sociedad, sean de carácter cultural, social, económico, político o tecnológico, que poseen relación directa en la construcción identitaria de los individuos. La perspectiva intercultural permearía ese proceso de construcción del material, de modo que el diálogo con el otro y/o entre culturas fuera un factor de negociación de las identidades en juego, tanto en clase como en la interacción investigador/profesor en formación. Estoy segura de que la unidad no podría abarcar la totalidad de asuntos que involucran el tema elegido, pero el profesor en formación puede realizar un recorte temático de manera que exponga su visión de mundo de aquel asunto y, a través de esa óptica, realizar el contraste necesario para la construcción de ideas, funcionando como punto de partida para una discusión crítica en clase.

Retornando al material, por el gran tema generador, cada profesor en formación eligió un tema individual para desarrollar una unidad didáctica del material de carácter intercultural. En este trabajo realizaré el análisis del tema migraciones desde la perspectiva de la categoría de análisis: Confrontación y diálogo con la cultura local. En esta categoría, analizo si la cultura local es tenida en consideración en la elaboración de la unidad didáctica. Al trabajar con la cultura del otro, el profesor precisa también relacionarla con la cultura local, en la cual sus alumnos están insertos, de manera que pueda ser establecido un diálogo en las diferencias y similitudes existentes.

La perspectiva intercultural en la enseñanza de lenguas presupone, entonces, una serie de acciones por el reconocimiento de la diversidad que nos constituye y del combate de las actitudes de discriminación con el otro. Para su concreción, todos los involucrados en el contexto escolar necesitan contribuir, y se hace necesario repensar la propia formación de los profesores, de manera que el currículum, los planes de enseñanza, planes de clase, materiales didácticos, evaluaciones y otros componentes del proceso pedagógico estén concebidos por una óptica que privilegie las actitudes críticas y de entendimiento entre las personas para la construcción de un mundo más justo. Como mi parte es la de la formación de profesores, me centro en las relaciones pasadas dentro de la escuela, pero la perspectiva intercultural también debe ser vista fuera 


\section{IIA Interculturalidad, educación y comunicación}

de ese ambiente, como una manera de vivir la vida, entendiendo cómo nos constituimos y como el otro se constituye. En el próximo apartado sigo con el análisis de la Unidad Didáctica propuesta para el trabajo con el tema 'migraciones'.

\section{El tema de las migraciones en confrontación y diálogo con la cultura local en la unidad didáctica propuesta}

El fenómeno de las migraciones ocupa un lugar destacado en el escenario mundial desde el inicio de nuestra civilización. Si pudiera establecer una relación, diría que la historia de las civilizaciones empieza con la historia de las migraciones humanas, pues el hombre, por diversas razones, necesitó migrar de una parte a la otra y, consecuentemente, se crearon espacios cada vez más multiétnicos, multiculturales y mestizos. En el siglo XXI las migraciones se acentúan, surgiendo nuevos problemas y la necesidad de comprender las situaciones generadas por ese fenómeno. De ese mosaico cultural surgen muchos conflictos de orden político, ideológico, religioso, étnico, cultural, entre otros, y no todos están preparados para vivir en este ambiente multicultural que se establece en nuestra sociedad. Buezas (2003: 35) apunta que la escuela posee un papel importante en este escenario:

«De ahí la necesidad de educar a todos en la solidaridad y tolerancia activa, particularmente con los inmigrantes. Pero para eso es necesario estar informados y conocer el fenómeno de la inmigración en sus dimensiones reales, entender cuáles son las razones por las cuales llegan a nuestras fronteras los inmigrantes, como fuimos anteriormente, evaluar los aspectos negativos que pueden llevar a un fenómeno tan complejo como la emigración; y encontrar las más adecuadas fórmulas para lograr una pacífica y justa convivencia entre todos, poniendo esfuerzo necesario de ambas las partes para conseguirlo»

El contexto que describo trae la inquietud sobre la capacidad de las escuelas en formar ciudadanos capaces de vivir en una sociedad con estas características. Para que se ofrezcan propuestas de resistencia a la tendencia al individualismo y a la exclusión de los más desfavorecidos es necesario que la perspectiva intercultural sea un elemento en la lucha contra una escuela y sociedad excluyentes, en la cual están incluidos los migrantes, sea cual sea su región de origen.

Pasamos al análisis de una parte de la unidad didáctica elaborada por una profesora en formación, con el tema de las migraciones, focalizándola categoría de análisis: Confrontación y diálogo con la cultura local. Destaco que la propuesta de esta investigación fue basada en el diálogo, o sea, el intercambio de experiencias fue la motivación para la interpretación de los datos obtenidos. En esta categoría de análisis, el discurso participativo de la profesora en formación reveló varios aspectos, algunos en la misma dirección y otros no. Sobre la experiencia vivida durante el proceso de investigación, la participante la vio positivamente y se mostró motivada, apuntando a la ampliación de sus conocimientos, la oportunidad de reflexión sobre la práctica, la posibilidad de entendimiento de las diferencias, entre otros.

La profesora en formación relató que como temática le gustaría trabajar la exclusión sufrida por migrantes nordestinos en la ciudad de São Paulo y sobre los principales problemas que enfrentan en las grandes ciudades de las regiones sureste y sur del país. Uno de los textos sugeridos para este tema fue la pintura Os retirantes de Cândido Portinari (disponible en: http:// 


\section{1/: El tema "migraciones" en materiales didácticos y la formación intercultural del profesorado de lengua española}

www.portinari.org.br/\#/acervo/conjunto/15).

Cândido Portinari fue un importante artista plástico brasileño, nacido en la ciudad Brodowski, São Paulo, en 1903 y fallecido en 1962. Es considerado uno de los artistas más prestigiados de Brasil y fue el pintor brasileño que alcanzó una mayor proyección internacional. Sus obras tienen gran interés social, retratando el brasileño común, buscando denunciar los problemas sociales del país. Una de las temáticas retratadas en sus obras fue la migración, como podemos ver en la pintura elegida por la profesora en formación.

Con esta actitud, el material partiría de una de las realidades del país, región de origen de los alumnos, para, a partir de ahí, iniciar la discusión sobre esta temática a nivel mundial, dando voz a culturas generalmente silenciadas en los manuales didácticos disponibles en el mercado, además de introducir un texto visual en clase. Teixeira (2008) indica que la lectura de una pintura o fotografía debe tener como principios, la contemplación y la concentración para que el observador se someta al impacto de las sensaciones. Al mencionar la pintura de Portinari, la autora apunta que:

«Porque se desvía de lo que existe como dado, de la semiótica natural y de la semiótica de la comunicación cotidiana, es que la pintura de Portinari, por ejemplo, mediante la exageración de pies y manos de los trabajadores o materializar geométricamente las lágrimas de los emigrantes crea un nuevo lenguaje que dramatiza y desestabiliza sentidos ya garantizados Ese nuevo lenguaje es materializado en recursos de expresión propios que atraen la atención del observador, aunque él no tenga conciencia de eso y salte directamente al contenido, a lo anecdótico de la pintura» (Teixeira, 2008: 303).

Sin embargo, en un encuentro posterior, ella relató que no iba más a utilizar la pintura mencionada por creer que estaría fuera de contexto, pues cree que solo sería posible si se hiciera un trabajo interdisciplinar con los profesores de historia y artes. A partir de esta constatación percibo que la profesora en formación desconoce la posibilidad de trabajar interdisciplinarmente dentro del ambiente de su clase, sin la necesidad de un proyecto mayor que integre otras disciplinas escolares. Uno de nuestros documentos oficiales, los Parámetros Curriculares Nacionales (PCN, 1998) defienden la enseñanza de manera interdisciplinar, a través de la integración de los temas transversales, que comprenden seis áreas: ética, orientación sexual, medio ambiente, salud, pluralidad cultural y trabajo y consumo.

Estos temas no constituyen asignaturas, sino discusiones que permean diversas áreas del conocimiento de manera transversal y actúan como un eje para que se pueda trabajar de manera contextualizada dentro de cada asignatura. Sabiendo que la lengua extranjera posee una función social en la escuela es interesante que los profesores sepan planear sus clases de forma que el significado sea construido y el aprendizaje tenga sentido, involucrando principalmente cuestiones de la realidad de los alumnos.

Percibí el interés por los textos no verbales y los de diferentes lenguajes, lo que es relevante por ofrecer otras lecturas, que merecen una inversión, en el sentido más amplio de la palabra, para ofrecer una variedad más allá del texto escrito. Al abrir espacio en el aula para la lectura de otros lenguajes, el profesor se aleja de la visión tradicional de la lectura de la palabra escrita como única posible y aumenta las posibilidades de ver el mundo por parte de los alumnos.

La profesora en formación no percibió que al elegir trabajar con pintura, es posible integrar las artes plás- 


\section{IIA Interculturalidad, educación y comunicación}

ticas en el aula de lengua extranjera y destacar el tema transversal pluralidad cultural. Paraquett (2004), al escribir un artículo sobre una propuesta de integración interdisciplinar con artes plásticas y enseñanza de lenguas extranjeras apunta que:

«La construcción de la identidad de una nación, como se sabe, se procesa a lo largo de su historia y, no podría ser diferente, se manifiesta a través de lenguajes. No solo de la lengua, sino a través de otras manifestaciones culturales como son, por ejemplo, las artes plásticas. Por eso, lengua, cultura, historia y artes plásticas son elementos indisociables en el aula de LE» (Paraquett, 2004: 196)

Así, la profesora en formación podría haber trabajado perfectamente con este género en su unidad didáctica, de manera que la pintura de Portinari pudiera levantar cuestiones sobre la migración en Brasil, denunciando problemas sociales que ocurrieron y aún ocurren en nuestro país. En este cuadro, Portinari expone el sufrimiento de muchos migrantes, representados visualmente por personas muy delgadas con expresiones que transmiten sentimientos de sufrimiento como el hambre y la miseria.

El cuadro fue creado en 1944, época en que muchas personas del nordeste migraban para el sur y sureste de Brasil, detrás de trabajo y mejores condiciones de vida, de donde podemos relacionar el nombre del cuadro -Retirantes- a los brasileños que vivieron/ viven en el nordeste de nuestro país y tuvieron la necesidad de abandonar su tierra en búsqueda de una vida mejor en otra parte del país. Paraquett (2004: 197) argumenta que el uso de la transversalidad como abordaje pedagógico "posibilita al alumno una visión amplia y consciente del mundo donde se inserta además de ser una forma de cuestionar la alienación y el individualismo en el conocimiento producido por la escuela".
Esta es una manera de oponerse a la fragmentación y a la linealidad del currículum escolar, siendo posible relacionar cuestiones de migración en nuestro país y de otros sitios en el mundo con la realidad de los alumnos de la escuela, ya que muchos pasaron o tuvieron familiares, amigos o conocidos que en algún momento de sus vidas tuvieron que migrar, no solamente por mejores condiciones de vida, como en la obra de Portinari, sino por otros motivos diversos.

De esa manera, también se fomenta una perspectiva intercultural, ya que conociendo al otro podemos conocernos mejor y entender aspectos históricos ocurridos en nuestro país en la comunidad que vivimos es una de las maneras de entender hechos de nuestro presente. El profesor tiene una función primordial en este proceso, pues él será el responsable de conducir las experiencias de clase, de manera que los alumnos puedan reflexionar sobre cuestiones que marcan la sociedad en que vivimos y confrontarlas con el otro a partir de nuestras experiencias puede fomentar la apertura a la diversidad.

De esa forma, la profesora en formación demuestra que cree que el trabajo interdisciplinar solo puede ser hecho en interacción con los otros profesores y no percibe la posible confrontación que enriquecería y partiría del conocimiento de mundo de los alumnos, valorando hechos históricos ocurridos en la cultura local.

Al preguntarle si buscó utilizar una perspectiva intercultural en su unidad didáctica y comentar las estrategias, la profesora en formación contestó que:

«Sí, pues busqué trabajar con textos provenientes de culturas diferentes, en el intento de trabajar cuestiones no solo culturales, sino las sociales y las individuales de los alumnos. Mi preocupación era siempre 


\section{1: El tema "migraciones" en materiales didácticos y la formación intercultural del profesorado de lengua española}

hacer que el alumno conociera la situación del otro, para reflexionar sobre su propia condición»

Por su respuesta, se percibe que hubo un intento de realizar la confrontación y diálogo con la cultura local, lo que es confirmado en varios momentos en el material y, por las discusiones ocurridas en los encuentros, percibo que ese intento realmente ocurrió, aunque algunas maneras de realizar este trabajo hayan sido descartadas. Ese puente (contexto del texto y contexto del alumno) debe ser hecho por el profesor, pues él será el responsable de conducir las experiencias en clase, para ayudar los alumnos a reflexionar sobre cuestiones que marcan la sociedad. El confronto con el otro a partir de nuestras experiencias puede y debe fomentar la apertura a la diversidad, ocasionando el diálogo intercultural, a partir de las diferencias y similitudes existentes.

\section{Consideraciones finales}

Como palabras finales sobre la Unidad Didáctica analizada, confirmé que para que los profesores elaboren una UD con la perspectiva intercultural es importante que, además de una formación basada en ese concepto, también reconozcan primeramente que la diversidad cultural está en el centro de todas las sociedades. De esa manera, los resultados me convencieron de la necesidad de construir acciones pedagógicas orientadas por una postura crítica, política, comprometida, en diálogo con las prácticas sociales y promoviendo el intercambio social.

Los resultados alcanzados apuntan la necesidad de fomentar desde el inicio de la titulación el interés por la investigación académica a través de proyectos que involucren a los futuros profesores con la rutina es- colar, lo que contribuye significativamente a la mejora de su práctica pedagógica. Sin embargo, para que se realicen prácticas pedagógicas con perspectivas interculturales, es necesario, antes de todo, conocer la realidad cultural de los alumnos(as) con los cuales se trabaja y, posteriormente elaborar un plan de curso que considere esa realidad, seleccionando, produciendo materiales y pensando en estrategias de evaluación del proceso (Mendes, 2007).

La concreción de esas prácticas será posible pensando en la formación de los profesores de lenguas extranjeras desde un prisma intercultural, pues serán ellos los responsables de poner en práctica y fomentar el diálogo entre culturas dentro de clase, y de esa manera, los Cursos de graduación en Letras con habilitación en una lengua extranjera necesitan reformular los planes político-pedagógicos y todo que tenga respeto a la formación de manera que la perspectiva intercultural esté presente en sus asignaturas y respectivos programas

Creo que en la formación inicial se debe reflexionar y teorizar sobre estas cuestiones, pues si el propio profesor posee sus estereotipos de lo que es una lenguacultura, ¿cómo podrá trabajar en el futuro y en clase para deconstruir las visiones reduccionistas de sus alumnos y promover un verdadero diálogo intercultural? Todos los profesores, sean universitarios o no, deben ser conscientes de su papel en esta sociedad, de cual es su papel político, para poder también exigir sus derechos y poder realizar un trabajo de calidad dentro de las universidades y escuelas: "Lo que nos compete es formar profesores para darles condiciones para ejercer con calidad y criticidad su trabajo" (Paraquett, 2009: 34).

Como educadora, entiendo que la enseñanza no pue- 


\section{IIA Interculturalidad, educación y comunicación}

de estar desvinculada de la realidad social, cultural y política para que no ocurra la reproducción de las desigualdades sociales y posibilite la realización de cambios donde no exista la convivencia pacífica entre las varias identidades a través del diálogo entre culturas. El enfoque intercultural es un medio para lograr eso en educación.

\section{Referencia}

Aguado, T. (2003). Pedagogía Intercultural. Madrid: McGraw-Hill.

Almeida Filho, J. C. P. de (2013). Codificar conteúdos, processo e reflexão formadora no material didático para ensino e aprendizagem de línguas, en Pereira, A. L. y Gottheim, L. (Orgs.). Materiais didáticos para o ensino de língua estrangeira: Processos de criação e contextos de uso, Campinas: Mercado de Letras.

Brasil (1998). Parâmetros Curriculares Nacionais: terceiro e quarto ciclos do ensino fundamental: língua estrangeira. Brasília, MEC/SEF.

Buezas, T. C. (2003). La escuela ante la inmigración y el racismo: Orientaciones de educación intercultural. Madrid: Editorial Popular.

Costa, E. G. de M. (2011). Lugar de aprender língua estrangeira é na escola: reflexões em torno do PNLD 2011. Letras, Santa Maria, v. 21, n. 42; 315-340.

Canclini, N. G. (2006). Culturas híbridas: estratégias para entrar e sair da modernidade. São Paulo: EDUSP. García M., Alfonso, Andrés E. F.y Andrés E. de H.(2007). La interculturalidad. Desafío para la educación. Madrid: Dykinson.

Mendes (2004). Abordagem Comunicativa Intercultural (ACIN): uma proposta para ensinar e aprender língua no diálogo de culturas. Tese (Doutorado em
Lingüística Aplicada) - Instituto de Estudos de Linguagens. Campinas: Universidade Estadual de Campinas.

Mendes, E. (2007). A perspectiva intercultural no ensino de línguas: uma relação "entre-culturas", en Alvarez, M. L. O. y Silva, K. A. (Orgs.). Lingüística Aplicada: múltiplos olhares. Campinas: Pontes.

Moita Lopes, L. P. (2003). Discursos de Identidades. Discurso como espaço de Construção de Gênero, Sexualidade, Raça, Idade e Profissão na Escola e na Família. Campinas: Mercado de Letras.

Paraquett, M. (2004). Uma integração interdisciplinar: artes plásticas e ensino de línguas estrangeiras, en Mota, K. y Schyerl, D. (Orgs.). Recortes interculturais: na sala de aula de línguas estrangeiras. Salvador: EDUFBA.

Paraquett, M. (2009). Lingüística Aplicada, inclusión social y aprendizaje de español en contexto latinoamericano. Revista Nebrija de Lingüística Aplicada, 6 (3), 1-23.

Rajagopalan, K. (2003). Por uma lingüística crítica: linguagem, identidade e a questão ética, São Paulo: Parábola.

Silva, M. B. da (2008). Novos horizontes no ensino de língua portuguesa: A formação do professor e o livro didático, en Mendes, E; Castro, M. L. Saberes em português: ensino e formação docente. Campinas: Pontes.

Teixeira, L. (2008). Leitura de textos visuais: princípios metodológicos, en Bastos, Neusa Barbosa (org.). Língua portuguesa: lusofonia - memória e diversidadecultural.São Paulo: EDUC. 